\title{
An IPHAS-based search for accreting very low-mass objects using VO tools
}

\author{
L. Valdivielso ${ }^{1}$, E. L. Martín ${ }^{1,2}$, H. Bouy ${ }^{1,3}$, E. Solano ${ }^{4,5}$, J. E. Drew ${ }^{6,7}$, \\ R. Greimel ${ }^{8,9}$, R. Gutiérrez ${ }^{4,5}$, Y. C. Unruh ${ }^{10}$, and J. S. Vink ${ }^{11}$ \\ 1 Instituto de Astrofísica de Canarias, C/ vía Láctea, s/n, 38200 La Laguna, Tenerife, Spain \\ e-mail: [lval; ege; bouy]@iac.es \\ 2 University of Central Florida, Dept. of Physics, PO Box 162385, Orlando, FL 32816-2385, USA \\ 3 Astronomy Department, University of California, Berkeley, CA 94720, USA \\ ${ }^{4}$ Laboratorio de Astrofísica Espacial y Física Fundamental (LAEFF-INTA), Apdo.78, 28691 Villanueva de la Cañada, Madrid, \\ Spain \\ e-mail: [esm;raul]@laeff.inta.es \\ 5 Spanish Virtual Observatory Thematic Network, Spain \\ ${ }^{6}$ Imperial College of Science, Technology and Medicine, Blackett Laboratory, Exhibition Road, London, SW7 2AZ, UK \\ e-mail: j.drew@imperial.ac.uk \\ 7 Centre for Astrophysics Research, University of Hertfordshire, College Lane, Hatfield AL10 9AB, UK \\ ${ }^{8}$ Institut für Physik, Universität Graz, Universitätsplatz 5, 8010 Graz, Austria \\ e-mail: rgreimel@gmail.com \\ 9 Isaac Newton Group of Telescopes, Apartado de correos 321, 38700 Santa Cruz de la Palma, Tenerife, Spain \\ 10 Astrophysics Group, Blackett Laboratory, Imperial College London, SW7 2AZ, UK \\ e-mail: y.unruh@imperial.ac.uk \\ 11 Armagh Observatory, College Hill, Armagh BT61 9DG, Northern Ireland, UK \\ e-mail: jsv@arm.ac.uk
}

Received 26 May 2008 / Accepted 12 October 2008

\section{ABSTRACT}

\begin{abstract}
Context. The main goal of this paper is to prove that accreting very low-mass stars and brown dwarfs can be identified in IPHAS, an $\mathrm{H} \alpha$ emission survey of the northern Milky Way. Full exploitation of the IPHAS database and a future extension of it in the southern hemisphere will be useful in identifying very low-mass accreting objects near to and far from well-known star-forming regions.

Aims. We have used Virtual Observatory tools to cross-match the IPHAS catalogue with the 2MASS catalogue. We defined photometric criteria to identify $\mathrm{H} \alpha$ emission sources with near-infrared colours similar to those of known young very low-mass stars and brown dwarfs. 4000 candidates were identified that met our criteria over an area of 1600 square degrees. We present low-resolution optical spectra of 113 candidates. Spectral types have been derived for the 33 candidates that have spectroscopically confirmed $\mathrm{H} \alpha$ emission, negligible reddening and spectral class $\mathrm{M}$. We have also measured $\mathrm{H} \alpha$ emission and investigated the NaI doublet $(818.3 \mathrm{~nm}, 819.5 \mathrm{~nm})$ in these 33 objects.

Methods. We confirm that 33 IPHAS candidates have strong $\mathrm{H} \alpha$ emission indicative of disc accretion for their spectral type. Twenty-three of them have spectral class M4 or later, of which ten have classes in the range M5.5-M7.0 and could thus be very young brown dwarfs. Many objects also have a weak NaI doublet, an indication of low surface gravity.

Results. IPHAS provides a very valuable database for identifying accreting very low-mass stars and brown dwarfs. Virtual Observatory tools provide an efficient method for identifying these objects over large areas of the sky. Based on our success rate of $23 \mathrm{H} \alpha$ emission objects with spectral type in the range M4-M7 out of 113 candidates with spectroscopic follow-up, we estimate that there could be hundreds of such objects in the full IPHAS survey.
\end{abstract}

Key words. stars: low-mass, brown dwarfs - stars: formation - stars: planetary systems: protoplanetary disks stars: pre-main sequence - infrared: stars

\section{Introduction}

Since the first unambiguous discovery of brown dwarfs (BDs) (Rebolo et al. 1995; Nakajima et al. 1995) this field has progressed rapidly. Considerable observational effort has been devoted to identifying BDs in known nearby star-forming regions (SFRs) and young open clusters. These objects provide crucial information on the dependence of key physical properties, such as disc properties, multiplicity and the shape of the initial mass function on primary mass.
Due to mass accretion processes, many young low-mass stars and BDs show stronger $\mathrm{H} \alpha$ emission than expected from chromospheric activity. Studying the $\mathrm{H} \alpha$ equivalent width and the spectral type using low-resolution spectra can determine whether or not a star is accreting (Barrado y Navascués \& Martín 2003). Thus $\mathrm{H} \alpha$ surveys have the potential to identify very young stars and BDs that are still accreting from their discs.

$\mathrm{H} \alpha$ searches for very low-mass (VLM) stars and BDs have already been instrumental in detecting these objects, such as those carried out in high latitude molecular clouds 
(Martin \& Kun 1996) and in Orion OB1 (Briceño et al. 2001). Accretion discs and infrared excess in young VLM stars and BDs have been confirmed in several SFRs, such as $\rho$ Ophiuchi (Wilking et al. 1999), the Trapezium (Muench et al. 2001), Chamaeleon I (Natta \& Testi 2001), IC 348 (Jayawardhana et al. 2003) and $\sigma$ Orionis (Oliveira et al. 2002; Zapatero Osorio et al. 2002a; Muzerolle et al. 2003). Some spectroscopic studies have emphasized analysis of the $\mathrm{H} \alpha$ emission of substellar objects and led to the detection of very cold objects such as S Ori 55 (Zapatero Osorio et al. 2002b) and S Ori 71 (Barrado y Navascués et al. 2002).

The INT Photometric $\mathrm{H} \alpha$ survey of the Northern Galactic Plane (IPHAS) is a valuable source for discovering young VLM stars and BDs using $\mathrm{H} \alpha$ emission. It covers 1800 square degrees of the northern Milky Way in the latitude range $-5^{\circ}<$ $b<5^{\circ}$. It provides (Sloan) $r^{\prime}, i^{\prime}$ and narrow-band $\mathrm{H} \alpha$ photometry down to a magnitude limit of $r^{\prime} \sim=20(10 \sigma)$. The data are taken using the Wide Field Camera (WFC) on the $2.5 \mathrm{~m}$ Isaac Newton Telescope (INT) (Drew et al. 2005; González-Solares et al. 2008).

So far, the overwhelming majority of the surveys for young VLM objects are concentrated in the known SFRs and nearby young clusters. Take as an example recent research in Taurus (Guieu et al. 2006), Orion OB1A and OB1B (Downes et al. 2006), Chamaeleon I (Luhman 2004a), Chamaeleon II and Ophiuchus (Allers et al. 2007) or $\sigma$ Orionis (Caballero et al. 2007). The IPHAS survey offers a complementary approach because it allows us to use $\mathrm{H} \alpha$ as a primary selection criterion and provides a wide area coverage around or outside the well-known SFRs and clusters.

The Virtual Observatory (VO) is a recent initiative with the goal of managing large databases in an organized manner in order to make efficient use of astronomical archives. In this work VO tools have been applied for the first time to the search for young VLM objects. We have made a cross-correlation of an early version of the IPHAS point source catalogue with the Two Micron All Sky Survey (2MASS) point source catalogue (Skrutskie et al. 2006). The rest of the paper is organized as follows: Sect. 2 describes the selection criteria used for identification of young VLM candidates. Section 3 deals with spectroscopic follow-up observations of 113 candidates selected using our criteria. Section 4 discusses the analysis of the spectra leading to the determination of spectral types and equivalent widths. Section 5 presents the main results of our study.

\section{Sample selection}

The VO offers the possibility of efficiently cross correlating large multi-wavelength databases. Aladin ${ }^{1}$ is an interactive software sky atlas allowing the user to visualize digitized astronomical images, superimpose entries from astronomical catalogues or databases, and interactively access related data and information from the Simbad database, the VizieR service and other archives for all known sources in the field.

We have used Aladin to look for new VLM objects via crosscorrelation of the IPHAS catalogue with 2MASS. The photometric data available in these catalogues provides magnitudes in $\mathrm{H} \alpha$, $r^{\prime}, i^{\prime}, J, H$ and $K$ for the selected objects.

We used an early version of the IPHAS point source catalogue that covered about 1200 square degrees. Our search

${ }^{1}$ http://aladin.u-strasbg.fr/aladin.gml was restricted to the RA range from 18 to $05 \mathrm{~h}$. We identified 4000 candidates that met the following criteria:

- IPHAS-2MASS coincidence in coordinates within 1";

- the IPHAS sources should be classified as stellar or probably stellar in the $r^{\prime}, i^{\prime}$ and $\mathrm{H} \alpha$ bands and have colours in the range $1.1<r^{\prime}-\mathrm{H} \alpha<3.0$ (to avoid potential artefacts and select objects with $\mathrm{H} \alpha$ in emission according to Drew et al. (2005), who show that M6 dwarfs have IPHAS colours $r^{\prime}-\mathrm{H} \alpha=$ 1.06 for $E(B-V)=0.0$, and $r-\mathrm{H} \alpha=1.14$ for $E(B-V)=$ 1.0); and magnitudes $i^{\prime}<18.5$;

- the 2MASS sources should have qflag A or B; colours in the range $0.7<J-H<1.3$ (to discard strong reddened objects and red giants); and $0.4<H-K<1.1$ (for selecting cool photospheres and/or infrared excess).

\section{Follow-up spectroscopic observations}

We have carried out three campaigns of follow-up low-resolution spectroscopic observation of our targets. Altogether, spectra for 113 candidates have been obtained, which represent only $3 \%$ of our total sample. We now discuss these runs in chronological order.

\subsection{William Herschel Telescope observations}

Our first run took place on 2006 August 1-2 with the ISIS long slit spectrograph on the $4.2 \mathrm{~m}$ William Herschel Telescope in La Palma as part of the international time programme led by Janet Drew. The R158R grating in the red arm was used. The instrumental setup gave a dispersion of $1.63 \AA$ /pixel with a wavelength range of 5400-10300 $\mathrm{A}$. We took spectra with exposure times ranging from 700 to $1800 \mathrm{~s}$ for 35 young VLM candidates. Because of poor weather conditions, on the first night (variable seeing of $\left.2-3^{\prime \prime}\right)$ the slit width was $2.0^{\prime \prime}$ (FWHM $\left.\sim 15 \AA\right)$. On the second night, we had much better seeing, ranging from $0.7^{\prime \prime}$ to $1^{\prime \prime}$; hence, a slit width of $1.0^{\prime \prime}$ was used (FWHM $7 \AA$ ). Each night, two flux calibration standard stars were observed, and $\mathrm{Ne}-\mathrm{Ar}$ arcs and lamp flat-fields were taken at the beginning of the nights. Bias and flat-field correction were applied to all our CCD frames. Wavelength calibration and instrument response corrections were made on the science spectra using standard tasks in the IRAF environment.

\subsection{Northern Optical Telescope observations}

Our second run occurred on 2006 October 10-14 with the ALFOSC spectrograph on the $2.5 \mathrm{~m}$ Nordic Optical Telescope in La Palma. Grism 5 was used, corresponding to a dispersion of $3.1 \AA /$ pixel and a wavelength range of 5000-10250 $\AA$. A slit width of $1.0^{\prime \prime}(F W H M \sim 16 \AA)$ was used and exposure times ranged from $300 \mathrm{~s}$ to $2400 \mathrm{~s}$. Fifty-six new candidates were observed. One flat-field and one arc lamp exposure were taken immediately before or after each science target. A flux calibration standard star was observed at the beginning of the nights. Bias correction was performed using an average bias for each night, and flat-field correction was made with the normalized flat-field exposure obtained at the same telescope position as the scientific spectrum. This procedure improved the correction of the fringing pattern in the CCD detector response over the correction that was obtained using an average flat-field. The calibration in wavelength was done using the images of $\mathrm{He}-\mathrm{Ne}-\mathrm{Th}-\mathrm{Ar}$ arcs obtained after each scientific image. Tests were done calibrating 
with the emission lines of the sky that appear in the spectra instead of with the arcs; nevertheless, the results of the calibration were not better. Tests were also made using different polynomial functions to fit the wavelength solution. Finally, a 4th-order Legendre polynomial was chosen. We measured the displacements of known sky airglow emission lines with respect to the expected positions to be generally less than $\sim 2 \AA$, which is acceptable because it is less than $1 / 8$ of our spectral resolution. The standard HD 227900 was used for flux calibrations. All data reductions were carried out using IRAF.

\subsection{Lick Observatory Shane Telescope observations}

In July 2007 we used the KAST spectrograph on the $3 \mathrm{~m}$ Shane Telescope at Lick Observatory with the 600/7500 (2.32 A/pixel) and 300/7500 (4.6 $\AA /$ pixel) gratings on the red arm only with a $2^{\prime \prime}$ slit width and exposure times from $300 \mathrm{~s}$ to $2400 \mathrm{~s}$ for a total of 25 candidates. The wavelength range covered was of 5600-8500 $\AA$ for the 600/7500 grating and 5000-10500 $\AA$ for the $300 / 7500$ grating. Neon arcs and flat-field lamp exposures were taken for each position of the scientific images. Part of the observations of the last night were affected by adverse weather (cirrus). In general the quality of the data collected during this campaign is poorer than on the two previous runs. During the first two nights, the 600/7500 grating was used ( $F W H M \sim 5 \AA$ ). Although the weather was good, with seeing of $\sim 1.5^{\prime \prime}$, the spectra were very noisy and weak, sometimes so much so that it was not possible to extract them. For the last two nights we decided to change to the $300 / 7500$ grating $(F W H M \sim 9 \AA)$ in order to improve the signal-to-noise ratio of the spectra. For the reduction, normalized flat-field images obtained after each scientific exposure was applied. For wavelength calibration Ne arcs, also taken after each exposure, were used. Depending on the night and the grating, different spectrophotometric standards were used for flux calibrations. All data reductions were carried out using IRAF.

\section{Spectral analysis}

We found that 42 of our 113 targets have strong $\mathrm{H} \alpha$ emission. We have therefore had a $37 \%$ success rate in the confirmation of $\mathrm{H} \alpha$ emission among our candidates. We find 12 of the 42 confirmed targets in the IPHAS catalogue of emission-line sources of Witham et al. (2008). For the sample selection we used a preliminary version of the IPHAS catalogue available to us in July 2006. Due to the improvement of the $\mathrm{H} \alpha$ zero point calibration on the IPHAS catalogue, although all of our candidates where selected as likely $\mathrm{H} \alpha$ emitters with $r^{\prime}-\mathrm{H} \alpha>1.1$, most of the non- $\mathrm{H} \alpha$ emitters are outside the main stellar locus of emission-line sources of Witham et al. (2008) in the IPHAS colour-colour plane, and only 48 of them fit the selection criteria according to the new photometric IPHAS catalogue. According to this, we have 48 observed candidates that really fit the criteria, and 40 of these show $\mathrm{H} \alpha$ emission which is an $83 \%$ success rate. In the following analysis we retain only those objects with confirmed $\mathrm{H} \alpha$ emission.

\subsection{Spectral type determination}

The spectral type has been determined using three different methods: (a) the Hammer code; (b) $\chi^{2}$ fitting with template spectra; and (c) measurement of spectral indices that have a known relation to spectral classification.

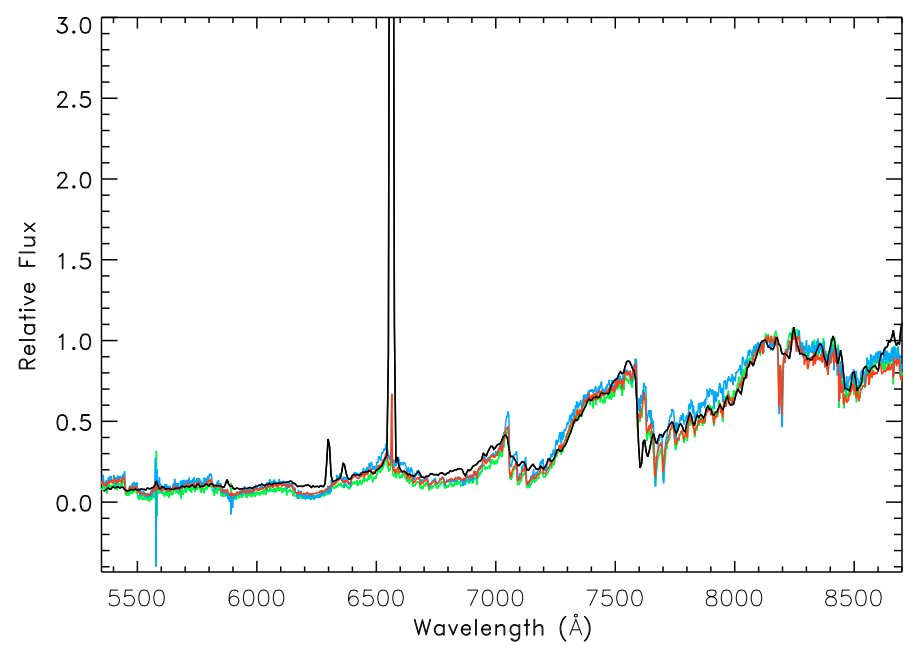

Fig. 1. An example of spectral type determination using the $\chi^{2}$ method. The best fit (M6) is represented in red overlaid on the observed spectrum J210404.87+535124.4 (black). For comparison we also show the M5 (blue) and M7 (green) reference objects. The spectra are normalized at $8123 \AA$.

\subsubsection{Comparison with template spectra: the Hammer code}

Hammer $^{2}$ is a tool written in IDL for the analysis of stellar spectra (Covey et al. 2007). With this routine each spectrum is analysed automatically to predict the spectral type by calculating a list of spectral indices (measuring the strength of $\mathrm{CaH}$ and $\mathrm{TiO}$ bands) to a library, considering the uncertainty of each index and also doing measurements of $\mathrm{H} \alpha$ as tracer of activity in late type stars. In addition, it allows the user to make manual comparisons and change the final spectral type that is initially assigned automatically by the program via a rough comparison with the sample of reference spectra. The routine has been applied to all our targets with confirmed emission in $\mathrm{H} \alpha$. In most cases, it was necessary to select the spectral type interactively. Nevertheless, applying this method is useful because it allows us quickly to inspect different comparisons of the targets with templates and to derive a first estimate of the spectral type.

\subsubsection{The $\chi^{2}$ method}

An IDL program has been written to calculate the $\chi^{2}$ value, comparing our target spectra with those of M-type reference field dwarfs. We found the best spectral type by minimization of the $\chi^{2}$ value. As reference for the spectral classification, stars of the IAC ultracool dwarf catalogue were used (Martín et al. 2005). We selected one dwarf for each spectral subclass between M0 and M9. The dwarfs selected were: SDSS J125350.99-001010.3 (M0), SDSS J004830.11-003029.1 (M1), SDSS J000201.55010636.5 (M2), SDSS J011113.97-003128.4 (M3), SDSS J002328.15+001709.02 (M4), SDSS J113922.08+000048.8 (M5), SDSS J150026.34-003928.0 (M6), SDSS J100218.61000408.46 (M7), SDSS J135859.02-005357.5 (M8) and SDSS J024958.36-003409.99 (M9). The spectra of the catalogue were resampled using the linterp task and normalized in the wavelength range of $\lambda=8110-8136 \AA$ (central wavelength $\lambda=$ $8123 \AA$ ) to do the calculation of $\chi^{2}$.

In Fig. 1 we display an example of the application of the $\chi^{2}$ method for the spectral type determination in one of our targets. The object spectrum (black line) is compared with the template

\footnotetext{
${ }^{2}$ See http://www.cfa.harvard. edu/ kcovey/thehammer
} 
Table 1. Spectral indices obtained for the IPHAS candidates.

\begin{tabular}{|c|c|c|c|c|}
\hline Object & PC3 & PC6 & TiO1 & $\mathrm{TiO} 2$ \\
\hline J001649.57+654241.8 & 1.20 & 9.50 & 1.52 & 1.22 \\
\hline \multirow{2}{*}{ J001655.91+654732.8 } & 1.23 & 8.08 & 1.55 & 1.19 \\
\hline & 1.16 & 7.68 & 1.44 & 1.10 \\
\hline $\mathrm{J} 011443.00+620820.9$ & 1.19 & 6.47 & 1.15 & 1.02 \\
\hline J012348.67+614931.8 & 1.13 & 4.01 & 1.21 & 1.05 \\
\hline $\mathrm{J} 013720.01+645957.7$ & 1.11 & 4.14 & 1.30 & 1.12 \\
\hline J023616.00+615609.6 & 0.99 & 2.54 & 1.17 & 0.94 \\
\hline J035449.17+530903.3 & 1.06 & 3.59 & 1.16 & 1.03 \\
\hline J035823.95+522312.6 & 1.00 & 2.01 & 1.03 & 0.88 \\
\hline J042450.68+455330.2 & 1.21 & 3.48 & 1.14 & 0.93 \\
\hline J183034.15+003800.6 & 1.35 & 7.46 & 1.22 & 0.92 \\
\hline $\mathrm{J} 183753.25+001849.2$ & 1.35 & 5.77 & 1.32 & 1.17 \\
\hline $\mathrm{J} 192656.04+211438.0$ & 1.05 & 1.76 & 1.03 & 0.96 \\
\hline $\mathrm{J} 202050.40+394243.6$ & 1.11 & 2.42 & 0.99 & 0.98 \\
\hline $\mathrm{J} 202434.30+422126.4$ & 1.06 & 6.91 & 1.66 & 1.29 \\
\hline J202437.28+385806.9 & 1.12 & 4.74 & 1.37 & 1.18 \\
\hline $\mathrm{J} 202455.53+424504.0$ & 1.12 & 4.94 & 1.56 & 1.31 \\
\hline J202759.82+390418.2 & 1.12 & 3.00 & 1.06 & 0.95 \\
\hline J204218.54+395723.8 & 1.00 & 2.77 & 1.04 & 0.99 \\
\hline $\mathrm{J} 204350.68+400108.8$ & 0.92 & 1.78 & 1.05 & 1.00 \\
\hline $\mathrm{J} 204704.82+434911.4$ & 1.08 & 2.12 & 1.05 & 0.97 \\
\hline $\mathrm{J} 205613.08+443424.2$ & 1.28 & 7.10 & 1.28 & 1.36 \\
\hline $\mathrm{J} 205701.63+434138.7$ & 1.28 & 8.90 & 1.75 & 1.46 \\
\hline J205702.69+434143.7 & 1.09 & 7.43 & 1.72 & 1.33 \\
\hline \multirow[t]{3}{*}{$\mathrm{J} 210404.87+535124.4$} & 1.43 & 7.95 & 1.26 & 1.14 \\
\hline & 1.17 & 7.09 & 1.45 & 1.22 \\
\hline & 1.30 & 7.59 & 1.47 & 1.27 \\
\hline $\mathrm{J} 213528.41+575823.0$ & 1.10 & 3.58 & 1.15 & 1.05 \\
\hline $\mathrm{J} 213545.87+573640.1$ & 1.09 & 2.97 & 1.23 & 1.01 \\
\hline \multirow[t]{2}{*}{$\mathrm{J} 213938.83+575451.4$} & 1.09 & 4.01 & 1.23 & 1.03 \\
\hline & 1.13 & 3.62 & 1.23 & 1.06 \\
\hline $\mathrm{J} 214547.73+564845.7$ & 1.29 & 6.43 & 1.42 & 1.22 \\
\hline \multirow[t]{2}{*}{$\mathrm{J} 214625.99+572829.0$} & 1.05 & 3.24 & 1.14 & 1.08 \\
\hline & 1.18 & 5.15 & 1.27 & 1.20 \\
\hline $\mathrm{J} 222025.22+605423.7$ & 1.11 & 3.11 & 1.24 & 1.03 \\
\hline \multirow[t]{2}{*}{$\mathrm{J} 224830.78+611417.9$} & 1.15 & 5.36 & 1.50 & 1.18 \\
\hline & 1.14 & 5.70 & 1.45 & 1.13 \\
\hline $\mathrm{J} 225331.29+623543.3$ & 1.26 & 8.62 & 1.36 & 1.06 \\
\hline $\mathrm{J} 225923.71+614138.6$ & 1.23 & 4.75 & 1.05 & 0.91 \\
\hline
\end{tabular}

that yields the minimum $\chi^{2}$ value (red line), and with the templates that have adjacent earlier or later spectral subclass (blue and green lines, respectively). In general the method works fine, although there were some objects that did not fit any spectral template properly. This method did not provide trustworthy results for noisy or highly reddened spectra because the calculation of $\chi^{2}$ is based on the difference point to point between two spectra.

\subsubsection{Spectral indices}

The PC3, PC6, TiO1 and TiO2 spectral indices of the objects with emission in $\mathrm{H} \alpha$ were determined using the IRAF sbands task and the results (see Table 1) were compared with those of Martín et al. (1999) to determine the spectral type of our candidates. For seven objects we could not find a reasonable agreement between the different indices (within two spectral subclasses), so this method was not used for them.

\subsection{Equivalent widths}

Hammer provides measurements of the equivalent width of $\mathrm{H} \alpha$ simultaneously with the spectral type determination. However,

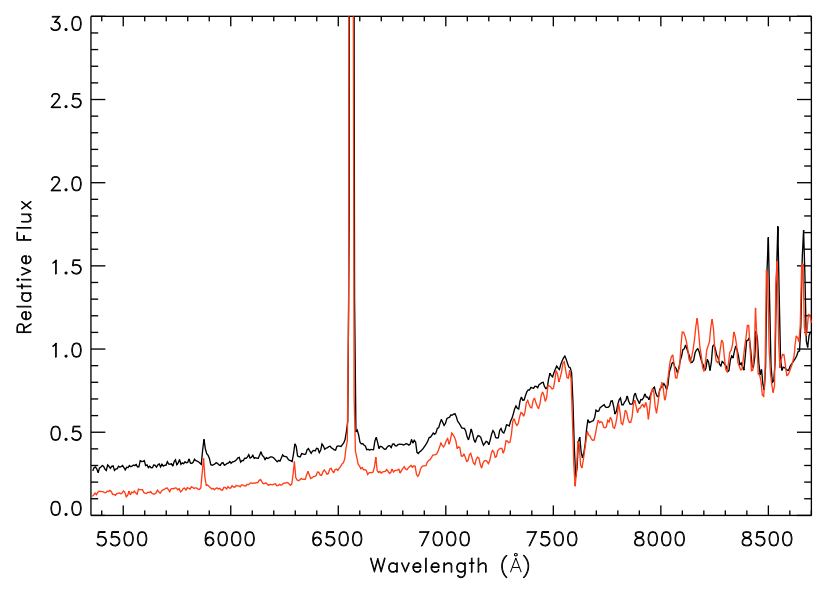

Fig. 2. Comparison of the spectra of IPHAS J214625.99+572829.0 observed on two different nights. There is significant variability in the continuum and the $\mathrm{H} \alpha$ emission. The spectra are normalized at $8123 \AA$.

it does not provide reliable errors for our spectra because it calculates them by propagating the error estimates provided by the SDSS spectral reduction pipeline.

We derived the $\mathrm{H} \alpha$ emission equivalent width of our targets by direct integration of the line profile using the IRAF splot task. The uncertainty was estimated via measurements performed for extreme choices of continuum level and line integration wavelength range made by visual inspection of the spectra.

We tried to measure the NaI doublet equivalent width at $818.3 \mathrm{~nm}$ and $819.5 \mathrm{~nm}$ in all of our objects as a low surface gravity indicator, but this part of the spectrum is affected by residual fringing and we cannot give a reliable estimation of the equivalent width. To investigate the surface gravity and for comparison purposes, simulations of how the residual fringing affects the doublet on the reference objects were carried out. We degraded the resolution of the reference objects to the resolution of our targets and introduced different residual fringing according to the residuals in our objects. For statistical and comparison purposes with our targets, and also for quantitative estimation of the variation with the residual fringing on the object, we used measurements of the $\mathrm{NaI}$ doublet index with defined blue, central and red bandpasses (8130-8171 $̊, 8172-8207 \AA$, 8235-8265 $\AA$ respectively due to low resolution of the spectra and visual inspection). The results are shown and discussed in Sect. 5.3.

\section{Results}

\subsection{Definition of the sample of M-type $\mathrm{H} \alpha$ emitters}

Nine of our 42 targets with confirmed $\mathrm{H} \alpha$ emission have very red continuum slopes on the red part of the spectrum, indicative of high reddening and are classified as early-M or late-K objects. They do not have late-M spectral type and are thus not considered in the rest of this paper. Our results are focused on the $33 \mathrm{M}$-type $\mathrm{H} \alpha$ emission objects that have negligible reddening according to our visual inspection of the comparison with reference dwarfs of known spectral types.

In Table 2 we give our results obtained from the three different methods of determination from the spectral type (SpT) and the measurements of equivalent widths $(W)$ of $\mathrm{H} \alpha$ (using Hammer and IRAF) and the Na I doublet.

Six of our targets were observed more than once to check for variability. They have more than one data line in Table 2. 
Table 2. Spectroscopic results.

\begin{tabular}{|c|c|c|c|c|c|c|}
\hline $\begin{array}{c}\text { IPHAS name/position } \\
\text { J[RA(2000)+Dec.(2000)] }\end{array}$ & $\begin{array}{c}\mathrm{SpT} \\
\text { (Hammer) }\end{array}$ & $\begin{array}{l}\mathrm{SpT} \\
\left(\chi^{2}\right)\end{array}$ & $\begin{array}{c}\text { SpT } \\
\text { (indices) }\end{array}$ & $\begin{array}{l}\text { SpT } \\
\text { (adopted) }\end{array}$ & $\begin{array}{l}\text { W(H } \alpha) \\
\text { (Hammer })\end{array}$ & $W(\mathrm{H} \alpha)$ \\
\hline J001649.57+654241.8 & M5 & M7 & M4.5 & M5.5 \pm 1.0 & 140.3 & $250 \pm 80$ \\
\hline \multirow[t]{2}{*}{ J001655.91+654732.8 } & M6 & M7 & M5.5 & M6 \pm 0.5 & 57.7 & $74 \pm 15$ \\
\hline & M5 & M7 & M5.5 & $\mathrm{M} 6 \pm 1.0$ & 96.1 & $133 \pm 15$ \\
\hline $\mathrm{J} 011443.00+620820.9$ & M4 & M5 & M4.5 & $\mathrm{M} 4.5 \pm 0.5$ & 192.7 & $276 \pm 50$ \\
\hline J012348.67+614931.8 & M4 & M4 & M4.5 & $\mathrm{M} 4 \pm 0.5$ & 135.6 & $230 \pm 30$ \\
\hline $\mathrm{J} 013720.01+645957.7$ & M4 & M4 & M4.5 & $\mathrm{M} 4 \pm 0.5$ & 112.7 & $170 \pm 30$ \\
\hline $\mathrm{J} 023616.00+615609.6$ & M1 & M4 & - & $\mathrm{M} 2.5 \pm 1.0$ & 133.7 & $205 \pm 30$ \\
\hline J035449.17+530903.3 & M3 & M4 & M2.5 & $\mathrm{M} 3 \pm 0.5$ & 93.7 & $164 \pm 30$ \\
\hline J035823.95+522312.6 & M1 & M2 & - & $\mathrm{M} 1.5 \pm 0.5$ & 147.2 & $214 \pm 30$ \\
\hline $\mathrm{J} 042450.68+455330.2$ & M3 & M4 & - & $\mathrm{M} 3.5 \pm 0.5$ & 21.6 & $122 \pm 30$ \\
\hline $\mathrm{J} 183034.15+003800.6$ & M6 & M8 & M5.5 & M6.5 \pm 1.0 & 185.8 & $161 \pm 50$ \\
\hline $\mathrm{J} 183753.25+001849.2$ & M6 & M7 & M5 & $\mathrm{M} 6 \pm 1.0$ & 16.3 & $94 \pm 30$ \\
\hline J192656.04+211438.0 & M0 & M0 & $M<2.5$ & $\mathrm{M} 0 \pm 0.5$ & 35.6 & $52 \pm 5$ \\
\hline $\mathrm{J} 202050.40+394243.6$ & M2 & M2 & M3 & $\mathrm{M} 2 \pm 0.5$ & 83.5 & $140 \pm 15$ \\
\hline $\mathrm{J} 202434.30+422126.4$ & M5 & M6 & M5.5 & M5.5 \pm 0.5 & 23.5 & $32 \pm 3$ \\
\hline J202437.28+385806.9 & M5 & M6 & M4.5 & M5 \pm 0.5 & 93.2 & $138 \pm 30$ \\
\hline $\mathrm{J} 202455.53+424504.0$ & M5 & M5 & M5 & M5 \pm 0.5 & 88.6 & $127 \pm 30$ \\
\hline J202759.82+390418.2 & M3 & M4 & - & M3.5 \pm 0.5 & 106.2 & $143 \pm 10$ \\
\hline $\mathrm{J} 204218.54+395723.8$ & M2 & M2 & - & $\mathrm{M} 2 \pm 0.5$ & 14.5 & $19 \pm 2$ \\
\hline $\mathrm{J} 204350.68+400108.8$ & M1 & M2 & - & M1.5 \pm 0.5 & 44.2 & $71 \pm 8$ \\
\hline $\mathrm{J} 204704.82+434911.4$ & M1 & M2 & M2.5 & $\mathrm{M} 2 \pm 0.5$ & 181.0 & $245 \pm 50$ \\
\hline $\mathrm{J} 205613.08+443424.2$ & M6 & M6 & M5 & M6 \pm 0.5 & 210.2 & $261 \pm 80$ \\
\hline J205701.63+434138.7 & M7 & M8 & M5.5 & $\mathrm{M} 7 \pm 1.0$ & 40.8 & $48.5 \pm 20$ \\
\hline $\mathrm{J} 205702.69+434143.7$ & M5 & M6 & M5.5 & M5.5 \pm 0.5 & 117.3 & $251 \pm 50$ \\
\hline \multirow[t]{3}{*}{$\mathrm{J} 210404.87+535124.4$} & M6 & M6 & M6 & M6 \pm 0.5 & 282.4 & $245 \pm 90$ \\
\hline & M6 & M6 & M5.5 & $\mathrm{M} 6 \pm 0.5$ & 288.6 & $580 \pm 100$ \\
\hline & M6 & M7 & M5.5 & M6 \pm 0.5 & 456.1 & $570 \pm 200$ \\
\hline $\mathrm{J} 213528.41+575823.0$ & M4 & M4 & M4.5 & $\mathrm{M} 4 \pm 0.5$ & 126.2 & $202 \pm 40$ \\
\hline $\mathrm{J} 213545.87+573640.1$ & M3 & M4 & M4 & $\mathrm{M} 4 \pm 0.5$ & 189.8 & $221 \pm 80$ \\
\hline \multirow[t]{2}{*}{$\mathrm{J} 213938.83+575451.4$} & M4 & M4 & M5 & M4.5 \pm 0.5 & 105.4 & $198 \pm 30$ \\
\hline & M4 & M4 & M4.5 & M4 \pm 0.5 & 124.0 & $166 \pm 40$ \\
\hline $\mathrm{J} 214547.73+564845.7$ & M4 & M6 & M5 & $\mathrm{M} 5 \pm 1.0$ & 147.8 & $131 \pm 55$ \\
\hline \multirow[t]{2}{*}{$\mathrm{J} 214625.99+572829.0$} & M2 & M3 & M2.5 & $\mathrm{M} 2.5 \pm 0.5$ & 243.8 & $360 \pm 60$ \\
\hline & M4 & M5 & M4.5 & M4.5 \pm 0.5 & 388.3 & $541 \pm 180$ \\
\hline $\mathrm{J} 222025.22+605423.7$ & M4 & M4 & M4.5 & $\mathrm{M} 4 \pm 0.5$ & 233.8 & $370 \pm 100$ \\
\hline \multirow[t]{2}{*}{ J224830.78+611417.9 } & M5 & M5 & M4.5 & M5 \pm 0.5 & 137.7 & $204 \pm 70$ \\
\hline & M5 & M5 & M5 & M5 \pm 0.5 & 143.7 & $190 \pm 30$ \\
\hline $\mathrm{J} 225331.29+623543.3$ & M6 & M7 & M5 & $\mathrm{M} 6 \pm 1.0$ & 194.6 & $350 \pm 100$ \\
\hline J225923.71+614138.6 & M5 & M5 & M5 & M5 \pm 0.5 & 250.1 & $540 \pm 50$ \\
\hline
\end{tabular}

We find that observations of the same objects obtained at different epochs and with different instruments give consistent spectral types within the error bars except for object IPHAS J214625.99+572829.0, which shows significant variability (Figs. 2 and 4). This object also displays strong and variable $\mathrm{H} \alpha$ emission. Two more objects (IPHAS J210404.87+535124.4 and $\mathrm{J} 001655.91+654732.8)$ show $\mathrm{H} \alpha$ variability larger than the error bars but no significant change in spectral type. The three remaining targets observed more than once do not have $\mathrm{H} \alpha$ variability larger than the error bars. These examples indicate that the observed $\mathrm{H} \alpha$ emission is not due to occasional flares because the strong $\mathrm{H} \alpha$ emission is observed at several epochs. Emissionline variability is an additional indicator for youth and has been found previously for young, accreting brown dwarfs (Scholz \& Jayawardhana 2006). The variability might be due to co-rotating hot spots, changes in the accretion flow geometry or changes in the mass accretion rate onto the central object (Bouvier et al. 1995).

In Fig. 3 the results of the three different methods of spectral type determination are compared. Hammer tends to give earlier types than the $\chi^{2}$ method. The discrepancies between these two methods is in general within one spectral subclass, but there are a few examples of larger differences. The spectral types obtained from the spectral indices are sometimes intermediate between Hammer and the $\chi^{2}$ method, but for the latest subclasses in our sample they tend to agree more with Hammer than with the $\chi^{2}$. We have adopted spectral subclasses by computing the average of the three methods, and we have rounded to the nearest subclass in steps of 0.5 subclass.

With respect to the measurements of the equivalent width of $\mathrm{H} \alpha$, it is observed that the values derived with Hammer are systematically lower than those obtained by manual integration of the line profile. We consider the latter to be more reliable than the Hammer results because they rely on careful visual inspection of the continuum and line profile in each spectrum. Hence, we adopt the manual values of $\mathrm{H} \alpha$ equivalent widths and error bars for the subsequent discussion.

\subsection{Disc accretion}

In order to discern whether the observed $\mathrm{H} \alpha$ emission is probably due to chromospheric activity or to disc accretion, we plot our $\mathrm{H} \alpha$ equivalent widths versus the adopted spectral type and compare these with the empirical upper limit boundary of 

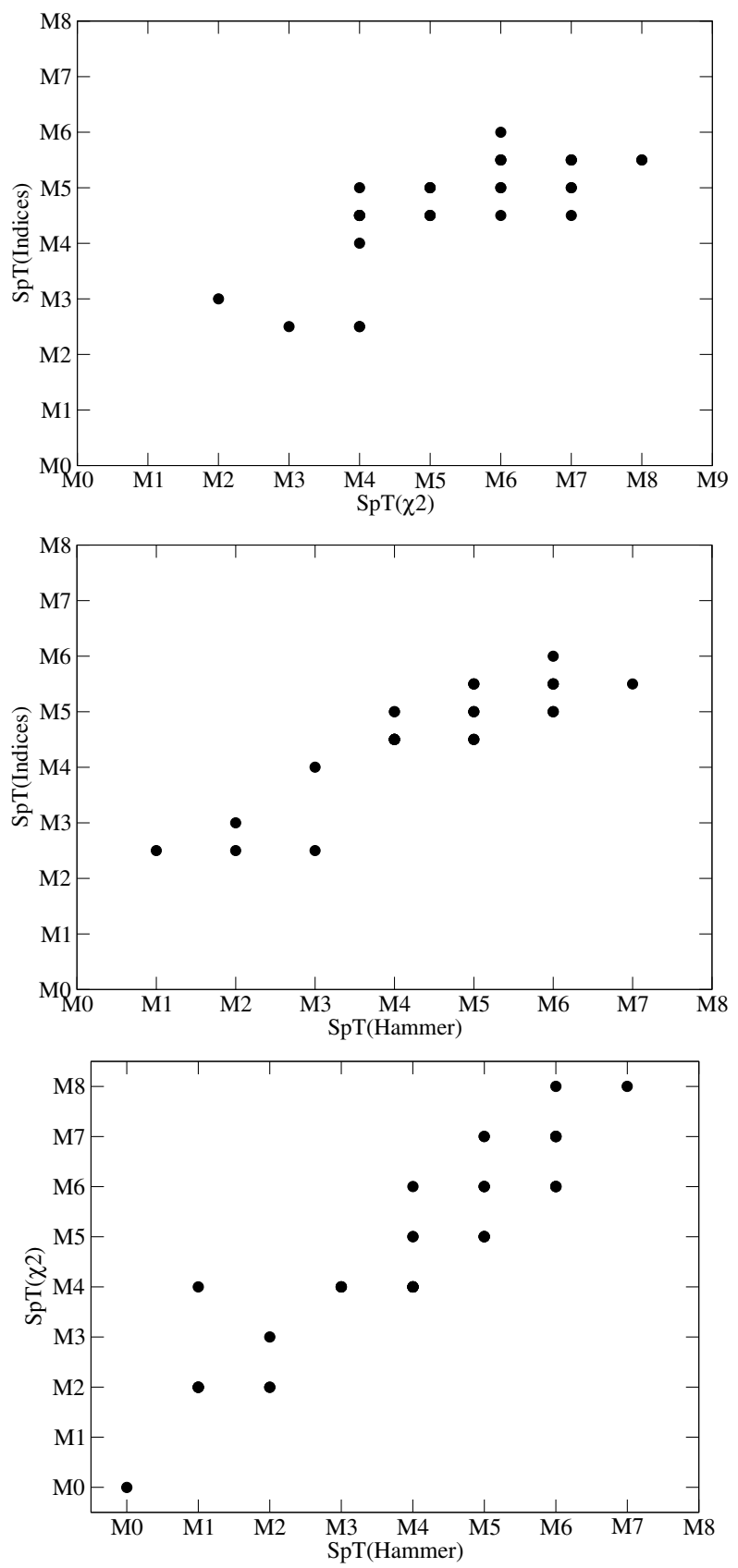

Fig. 3. Comparison of spectral type determinations using different methods.

chromospheric activity derived by Barrado y Navascués \& Martín (2003). The results are shown in Fig. 4. The six objects observed more than once are marked with red squares and the different measurements are connected with red lines. As mentioned in the previous section, the persistence of the strong $\mathrm{H} \alpha$ emission indicates that it is not due to sporadic flares. All the objects occupy the region of the diagram above the chromospheric activity saturation line and are thus probably accreting matter from discs.

\subsection{Surface gravity}

The NaI subordinate doublet at $818.3 \mathrm{~nm}$ and $819.5 \mathrm{~nm}$ is a good gravity indicator for late-M spectral types (Kirkpatrick et al. 1991; Martin et al. 1996; Briceño et al. 1998). This doublet becomes weaker as the surface gravity decreases. We have checked

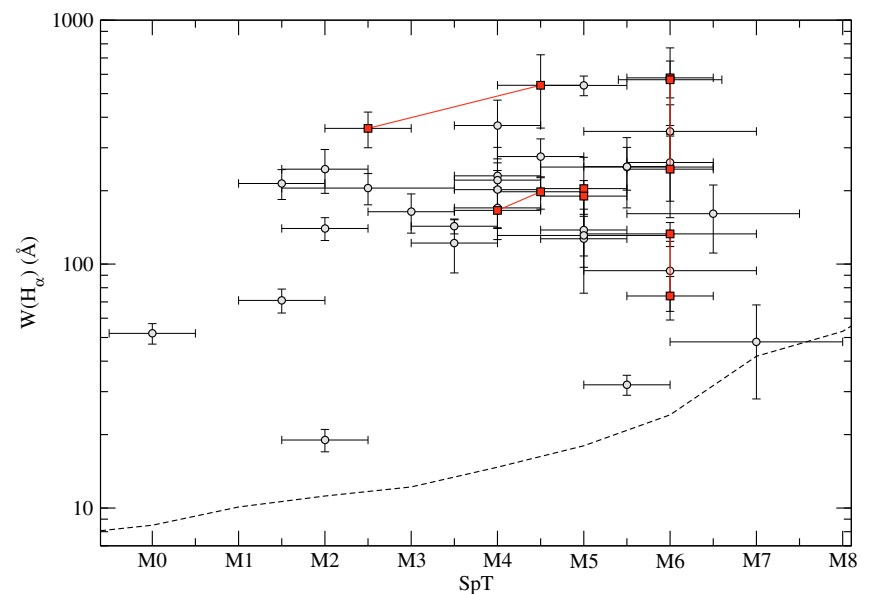

Fig. 4. $\mathrm{H} \alpha$ equivalent width against adopted spectral type for our objects. The dashed line denotes the dividing line between chromospheric activity and disc accretion. Our objects are clearly above the dashed line and are hence probably undergoing mass accretion.

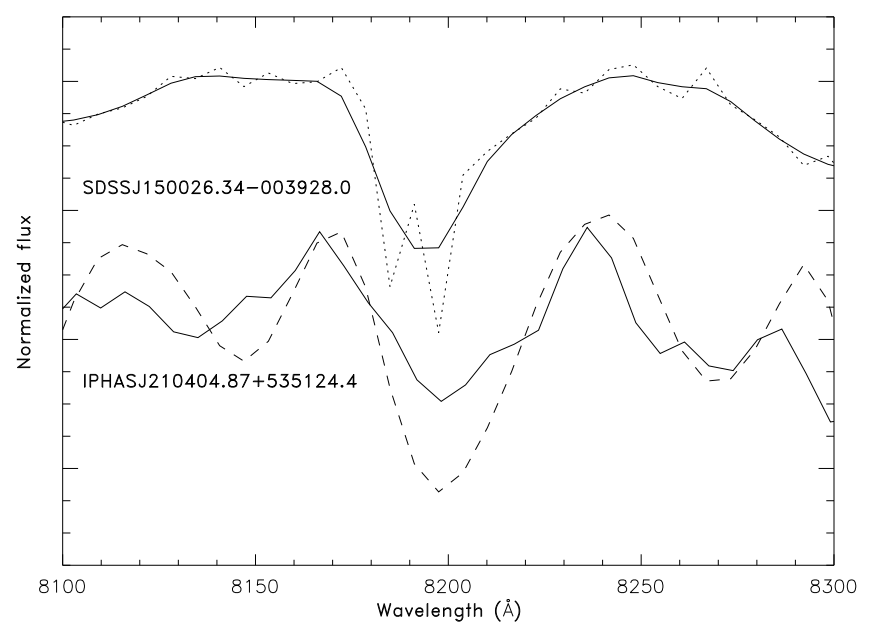

Fig. 5. Effects of the spectral resolution and residual fringing on our M6 template (SDSSJ150026.34-003928.0). Dotted line: original spectrum of the template; solid line: the same spectrum degraded to our spectral resolution; dashed line: the same spectrum degraded to our spectral resolution, and with residual fringing. For comparison we also show the spectrum of a typical M6 object from our sample (IPHAS J210404.87+535124.4).

whether the doublet can be identified in the reference objects despite the fringing and low resolution of our targets. In Fig. 5 we compare the SDSSJ150026.34-003928.0 reference dwarf (solid and dotted top lines and dashed bottom line) with one of our M6 classified objects IPHAS J210404.87+535124.4 (bottom solid line) and the doublet can be clearly identified despite the residual fringing and low resolution. In Fig. 6 we show the results of the measurements described in Sect. 4.2. We find that for M3 type and later the doublet can be clearly identified but not for earlier types since the doublet is weaker for these spectral types and is embedded in the residual fringing. According to these results, we may conclude that in all of our objects classified as M3-4 the NaI doublet is weaker in our $\mathrm{H} \alpha$ emission objects than in field dwarfs of similar spectral class and much weaker for objects later than M4. This is consistent with our previous conclusion that most of our objects are very young and actively accreting. The half-life for disc accretion in VLM objects has been estimated at less than 5 Myr by Mohanty et al. (2005). 
Table 3. Photometric data for the IPHAS objects.

\begin{tabular}{|c|c|c|c|c|c|c|c|}
\hline \multirow{2}{*}{$\begin{array}{c}\text { IPHAS name/position } \\
J[\mathrm{RA}(2000)+\text { Dec. }(2000)]\end{array}$} & \multirow{2}{*}{$\begin{array}{c}\text { Observation } \\
\text { date }\end{array}$} & \multicolumn{3}{|c|}{ IPHAS magnitudes } & \multicolumn{3}{|c|}{ 2MASS magnitudes } \\
\hline & & $r^{\prime}$ & $r^{\prime}-i^{\prime}$ & $r^{\prime}-\mathrm{H} \alpha$ & $J$ & $H$ & K \\
\hline J001649.57+654241.8 & $2004-08-07$ & $20.308 \pm 0.045$ & $2.015 \pm 0.054$ & $2.107 \pm 0.050$ & $15.005 \pm 0.053$ & $13.758 \pm 0.052$ & $12.981 \pm 0.041$ \\
\hline J001655.91+654732.8 & 2004-08-07 & $18.803 \pm 0.014$ & $2.209 \pm 0.017$ & $1.593 \pm 0.018$ & $13.645 \pm 0.026$ & $12.506 \pm 0.023$ & $11.993 \pm 0.022$ \\
\hline J011443.00+620820.9 & $2003-10-10$ & $20.033 \pm 0.148$ & $1.756 \pm 0.155$ & $2.115 \pm 0.153$ & $15.487 \pm 0.064$ & $14.198 \pm 0.070$ & $13.411 \pm 0.042$ \\
\hline J012348.67+614931.8 & $2003-10-12$ & $19.716 \pm 0.081$ & $1.722 \pm 0.086$ & $2.102 \pm 0.083$ & $15.636 \pm 0.061$ & $14.541 \pm 0.060$ & $14.039 \pm 0.053$ \\
\hline J013720.01+645957.7 & $2003-10-14$ & $18.001 \pm 0.028$ & $1.721 \pm 0.030$ & $1.318 \pm 0.031$ & $13.621 \pm 0.028$ & $12.687 \pm 0.036$ & $12.083 \pm 0.023$ \\
\hline J023616.00+615609.6 & 2003-11-10 & $19.727 \pm 0.087$ & $1.280 \pm 0.096$ & $2.041 \pm 0.090$ & $15.984 \pm 0.099$ & $15.022 \pm 0.103$ & $14.285 \pm 0.076$ \\
\hline J035449.17+530903.3 & 2004-12-01 & $20.249 \pm 0.059$ & $1.961 \pm 0.071$ & $1.906 \pm 0.072$ & $15.719 \pm 0.087$ & $14.719 \pm 0.088$ & $14.055 \pm 0.072$ \\
\hline J035823.95+522312.6 & $2004-12-29$ & $19.016 \pm 0.038$ & $1.283 \pm 0.057$ & $1.965 \pm 0.042$ & $15.745 \pm 0.061$ & $14.826 \pm 0.061$ & $14.298 \pm 0.064$ \\
\hline J042450.68+455330.2 & $2004-10-22$ & $18.619 \pm 0.017$ & $1.732 \pm 0.021$ & $1.662 \pm 0.021$ & $14.116 \pm 0.030$ & $12.76 \pm 0.026$ & $11.849 \pm 0.022$ \\
\hline $\mathrm{J} 183034.15+003800.6$ & 2004-06-08 & $18.368 \pm 0.010$ & $1.907 \pm 0.013$ & $1.983 \pm 0.012$ & $13.633 \pm 0.028$ & $12.524 \pm 0.031$ & $11.937 \pm 0.024$ \\
\hline $\mathrm{J} 183753.25+001849.2$ & 2004-06-09 & $19.134 \pm 0.018$ & $2.621 \pm 0.021$ & $1.566 \pm 0.024$ & $13.05 \pm 0.026$ & $11.837 \pm 0.021$ & $11.16 \pm 0.022$ \\
\hline J192656.04+211438.0 & 2004-08-06 & $16.667 \pm 0.005$ & $1.195 \pm 0.008$ & $1.313 \pm 0.007$ & $12.347 \pm 0.022$ & $11.39 \pm 0.021$ & $10.742 \pm 0.019$ \\
\hline $\mathrm{J} 202050.40+394243.6$ & $2007-06-21$ & $19.954 \pm 0.037$ & $1.110 \pm 0.056$ & $1.757 \pm 0.049$ & $15.83 \pm 0.076$ & $14.569 \pm 0.051$ & $13.611 \pm 0.050$ \\
\hline $\mathrm{J} 202434.30+422126.4$ & $2003-08-10$ & $18.304 \pm 0.040$ & $1.912 \pm 0.042$ & $1.170 \pm 0.046$ & $13.335 \pm 0.024$ & $12.249 \pm 0.027$ & $11.501 \pm 0.023$ \\
\hline J202437.28+385806.9 & 2006-11-04 & $19.879 \pm 0.070$ & $1.921 \pm 0.078$ & $1.664 \pm 0.080$ & $15.337 \pm 0.055$ & $14.293 \pm 0.052$ & $13.685 \pm 0.055$ \\
\hline $\mathrm{J} 202455.53+424504.0$ & 2004-08-08 & $18.902 \pm 0.014$ & $1.910 \pm 0.018$ & $1.525 \pm 0.020$ & $14.425 \pm 0.037$ & $13.548 \pm 0.038$ & $12.883 \pm 0.030$ \\
\hline $\mathrm{J} 202759.82+390418.2$ & $2006-11-24$ & $17.947 \pm 0.013$ & $1.450 \pm 0.017$ & $1.820 \pm 0.015$ & $13.729 \pm 0.030$ & $12.603 \pm 0.026$ & $11.897 \pm 0.022$ \\
\hline J204218.54+395723.8 & 2007-06-29 & $17.712 \pm 0.009$ & $1.468 \pm 0.012$ & $0.729 \pm 0.015$ & $14.152 \pm 0.028$ & $13.099 \pm 0.029$ & $12.587 \pm 0.026$ \\
\hline $\mathrm{J} 204350.68+400108.8$ & $2007-06-29$ & $17.273 \pm 0.007$ & $1.270 \pm 0.010$ & $1.223 \pm 0.010$ & $14.035 \pm 0.031$ & $13.024 \pm 0.023$ & $12.44 \pm 0.026$ \\
\hline $\mathrm{J} 204704.82+434911.4$ & $2003-10-12$ & $18.758 \pm 0.032$ & $1.232 \pm 0.037$ & $2.049 \pm 0.034$ & $15.336 \pm 0.054$ & $14.343 \pm 0.051$ & $13.764 \pm 0.043$ \\
\hline J205613.08+443424.2 & 2003-11-10 & $20.250 \pm 0.113$ & $1.958 \pm 0.121$ & $2.267 \pm 0.120$ & $14.678 \pm 0.043$ & $13.895 \pm 0.056$ & $13.387 \pm 0.045$ \\
\hline $\mathrm{J} 205701.63+434138.7$ & $2007-06-23$ & $18.764 \pm 0.013$ & $2.269 \pm 0.016$ & $1.373 \pm 0.019$ & $13.952 \pm 0.029$ & $13.142 \pm 0.032$ & $12.649 \pm 0.029$ \\
\hline J205702.69+434143.6 & $2007-06-23$ & $19.871 \pm 0.029$ & $2.252 \pm 0.034$ & $1.868 \pm 0.036$ & $14.348 \pm 0.055$ & $13.382 \pm 0.041$ & $12.695 \pm 0.039$ \\
\hline $\mathrm{J} 210404.87+535124.4$ & 2006-10-02 & $18.299 \pm 0.015$ & $1.464 \pm 0.020$ & $2.507 \pm 0.016$ & $13.432 \pm 0.025$ & $12.401 \pm 0.025$ & $11.613 \pm 0.025$ \\
\hline $\mathrm{J} 213528.41+575823.0$ & 2004-08-05 & $18.640 \pm 0.015$ & $1.360 \pm 0.025$ & $1.980 \pm 0.019$ & $15.15 \pm 0.059$ & $14.212 \pm 0.056$ & $13.598 \pm 0.044$ \\
\hline $\mathrm{J} 213545.87+573640.1$ & 2004-08-05 & $18.526 \pm 0.014$ & $1.509 \pm 0.021$ & $1.996 \pm 0.017$ & $14.551 \pm 0.037$ & $13.676 \pm 0.034$ & $13.143 \pm 0.033$ \\
\hline J213938.83+575451.4 & $2004-08-27$ & $19.637 \pm 0.044$ & $1.658 \pm 0.053$ & $2.083 \pm 0.049$ & $14.757 \pm 0.039$ & $13.621 \pm 0.040$ & $13.032 \pm 0.033$ \\
\hline $\mathrm{J} 214547.73+564845.7$ & $2004-08-28$ & $18.942 \pm 0.035$ & $2.058 \pm 0.039$ & $1.740 \pm 0.040$ & $14.333 \pm 0.032$ & $13.399 \pm 0.036$ & $12.822 \pm 0.025$ \\
\hline $\mathrm{J} 214625.99+572829.0$ & 2004-08-29 & $18.592 \pm 0.036$ & $1.377 \pm 0.046$ & $2.315 \pm 0.038$ & $14.069 \pm 0.034$ & $13.064 \pm 0.033$ & $12.301 \pm 0.026$ \\
\hline $\mathrm{J} 222025.22+605423.7$ & 2003-11-03 & $19.167 \pm 0.051$ & $1.459 \pm 0.057$ & $2.130 \pm 0.053$ & $15.32 \pm 0.053$ & $14.413 \pm 0.051$ & $13.911 \pm 0.052$ \\
\hline $\mathrm{J} 224830.78+611417.9$ & 2004-08-05 & $19.650 \pm 0.034$ & $2.069 \pm 0.040$ & $1.988 \pm 0.040$ & $14.959 \pm 0.043$ & $13.967 \pm 0.040$ & $13.458 \pm 0.036$ \\
\hline $\mathrm{J} 225331.29+623543.3$ & 2004-08-21 & $20.554 \pm 0.051$ & $2.153 \pm 0.062$ & $1.997 \pm 0.060$ & $15.393 \pm 0.059$ & $14.17 \pm 0.046$ & $13.471 \pm 0.035$ \\
\hline J225923.71+614138.6 & $2007-06-27$ & $19.228 \pm 0.016$ & $1.493 \pm 0.023$ & $2.219 \pm 0.019$ & $15.341 \pm 0.071$ & $14.192 \pm 0.057$ & $13.487 \pm 0.051$ \\
\hline
\end{tabular}

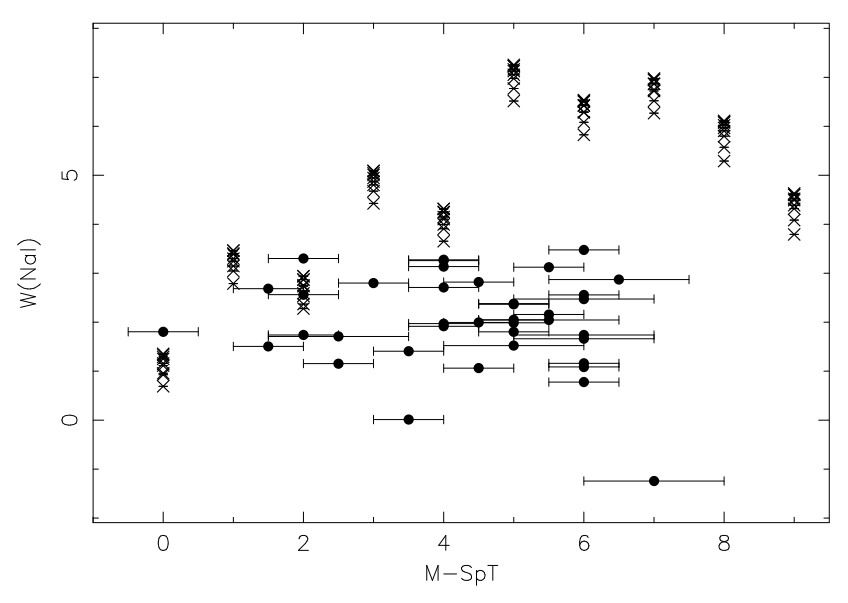

Fig. 6. Dependence of $W(\mathrm{Na} \mathrm{I})$ on residual fringing and spectral type. Filled circles denote the IPHAS objects and crosses denote measurements with different fringing residuals for the $\mathrm{M}$ objects used as reference to classify the IPHAS objects.

According to those authors, the results suggest that the half-life of our sample is $\leq 5 \mathrm{Myr}$.

\subsection{Photometric properties}

The main emphasis of our analysis has been on the spectroscopic properties of our sample. However, it is also interesting to study the photometric properties. The photometry, according to the best new IPHAS photometry of our 33 main objects, is given in Table 3. Colour-magnitude (Fig. 7) and colour-colour (Fig. 8) diagrams are used to check whether our sample of 33 interesting objects stand out from the crowd.

In Fig. 7 we have represented by a plus sign the objects classified as M4 or later. It is observed that these tend to populate the fainter part of the diagram. All of them have magnitudes of $r^{\prime}>16.5, J>12, i^{\prime}>15.5$ and $H>11$.

In Fig. 8, we also plot as open triangles the objects that show emission in $\mathrm{H} \alpha$ but are classified as earlier than M4 and those classified as M4 or later with plus signs. All the accretors show red colours with $H-K>0.5$, and most of them $i^{\prime}-J<4$. We find that the $r^{\prime}-i^{\prime}$ and $r^{\prime}-H$ colours have a blueward tendency, which could be related to the presence of optical veiling in the $r^{\prime}$ band. On the other hand, the red $H-K$ colours could be ascribed to warm dust emission.

We have compared the colour-spectral type relation for our targets classified as M4 or later with those in the study of Hawley et al. (2002) in which the spectroscopic characterization of SDSS M dwarfs was performed (see Fig. 9). In general our objects follow the relation observed in the SDSS objects, but there are some significant discrepancies. In the $r^{\prime}-i^{\prime}$ vs. SpT diagram, a tendency to the blue for some objects compared with SDSS locus is observed. On the other hand, for the $i^{\prime}-J$ colour, a tendency to the red is observed for our sample. These discrepancies could be due to the effects of accretion and low surface gravity on the spectral energy distribution of VLM objects. 

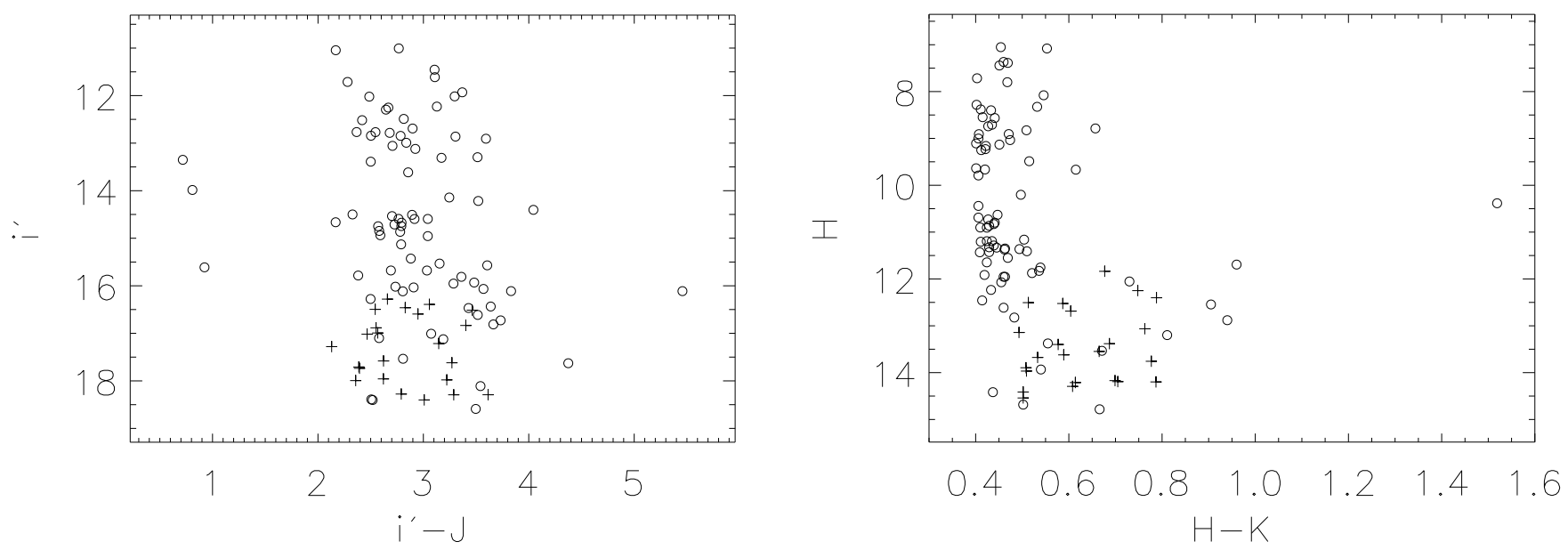

Fig. 7. Colour-magnitude diagrams of the candidates. Open circles represent the objects observed spectroscopically; plus signs identify the observed objects that show $\mathrm{H} \alpha$ emission and are classified as M4 or later.
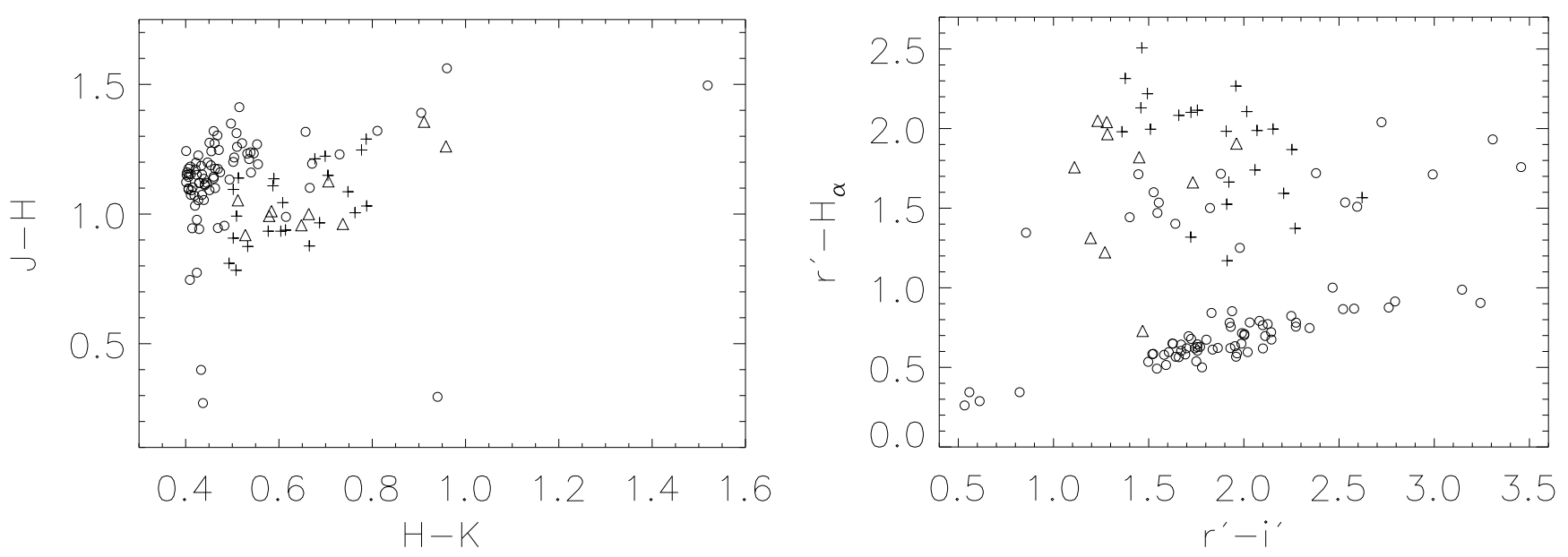

Fig. 8. Colour-colour diagrams. Open circles represent all objects observed spectroscopically; open triangles represent the objects that show $\mathrm{H} \alpha$ emission and are classified as earlier than M4, and plus signs represent the objects that show $\mathrm{H} \alpha$ emission and are classified as M4 or later.
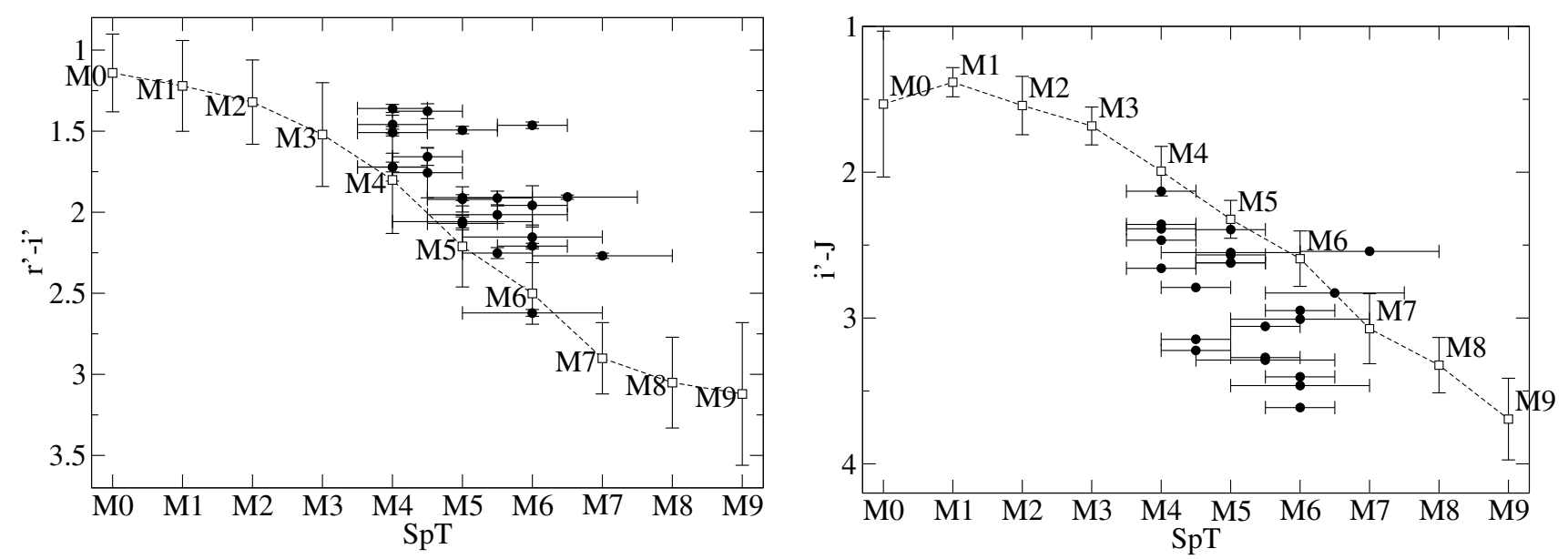

Fig. 9. Comparison of the results for the IPHAS objects (filled circles) and the characterization for M dwarfs of Hawley et al. (2002) (open squares).

Acknowledgements. Part of the data presented here were taken using ALFOSC, which is owned by the Instituto de Astrofísica de Andalucía (IAA) and operated at the Nordic Optical Telescope under agreement between the IAA and the NBIfAFG of the Astronomical Observatory of Copenhagen. This paper makes use of data obtained as part of the INT Photometric $\mathrm{H} \alpha$ Survey of the Northern Galactic Plane (IPHAS) carried out at the Isaac Newton Telescope. The INT and
WHT are operated on the island of La Palma by the Isaac Newton Group in the Spanish Observatorio del Roque de los Muchachos of the Instituto de Astrofísica de Canarias. All IPHAS data are processed by the Cambridge Astronomical Survey Unit, at the Institute of Astronomy in Cambridge. This publication makes use of data products from the Two Micron All Sky Survey, which is a joint project of the University of Massachusetts. This work makes use of EURO-VO software, 
tools or services. The EURO-VO has been funded by the European Commission through contract numbers RI031675 (DCA) and 011892 (VO-TECH) under the 6th Framework Programme. This research has made use of the Spanish Virtual Observatory supported from the Spanish MEC through grants AyA2005-04286, AyA2005-24102-E. This project has also been supported by MEC, through grant AyA2007-67458.

\section{References}

Allers, K. N., Jaffe, D. T., Luhman, K. L., et al. 2007, ApJ, 657, 511 Barrado y Navascués, D., Zapatero Osorio, M. R., Martín, E. L., et al. 2002, A\&A, 393, L85

Barrado y Navascués, D., \& Martín, E. L. 2003, AJ, 126, 2997

Bouvier, J., Covino, E., \& Kovo, et al. 1995, A\&A, 299, 89

Briceño, C., Hartmann, L., Stauffer, J., \& Martín, E. 1998, AJ, 115, 2074

Briceño, C., Vivas, A., Katherina, C. N., et al. 2001, Science, 291, 93

Caballero, J. A., Béjar, V. J. S., Rebolo, R., et al. 2007, A\&A, 470, 903

Covey, K. R., Ivezić, Ž., Schlegel, D., et al. 2007, AJ, 134, 2398

Downes, J. J., Briceño, C., \& Hernández, J. 2006, Rev. Mex. Astron. Astrofis. Conf. Ser., 26, 37

Drew, J. E., Greimel, R., Irwin, M. J., et al. 2005, MNRAS, 362, 753

González-Solares, E. A., Walton, N. A., Greimel, R., et al. 2008, MNRAS, 388, 89

Guieu, S., Dougados, C., Monin, J.-L., et al. 2006, A\&A, 446, 485
Hawley, S. L., Covey, K. R., Knapp, G. R., et al. 2002, AJ, 123, 3409 Jayawardhana, R., Mohanty, S., \& Basri, G. 2003, ApJ, 592, 282 Kirkpatrick, J. D., Henry, T. J., \& McCarthy, D. W., Jr. 1991, ApJS, 77, 417

Luhman, K. L. 2004a, ApJ, 602, 816

Luhman, K. L. 2004b, ApJ, 614, 398

Martin, E. L., \& Kun, M. 1996, A\&AS, 116, 467

Martin, E. L., Rebolo, R., \& Zapatero-Osorio, M. R. 1996, ApJ, 469, 706

Martín, E. L., Delfosse, X., Basri, G., et al. 1999, AJ, 118, 2466

Martín, E. L., Cabrera, J., \& Cenizo, E. 2005, Astron. Nachr., 326, 1026

Mohanty, S., Jayawardhana, R., \& Basri, G. 2005, ApJ, 626, 498

Muench, A. A., Alves, J., Lada, C. J., \& Lada, E. A. 2001, ApJ, 558, L51

Muzerolle, J., Hillenbrand, L., Calvet, N., Briceño, C., \& Hartmann, L. 2003, ApJ, 592, 266

Nakajima, T., Oppenheimer, B. R., Kulkarni, S. R., et al. 1995, Nature, 378, 463 Natta, A., \& Testi, L. 2001, A\&A, 376, L22

Oliveira, J. M., Jeffries, R. D., Kenyon, M. J., Thompson, S. A., \& Naylor, T. 2002, A\&A, 382, L22

Rebolo, R., Zapatero-Osorio, M. R., \& Martin, E. L. 1995, Nature, 377, 129

Scholz, A., \& Jayawardhana, R. 2006, ApJ, 638, 1056

Skrutskie, M. F., Cutri, R. M., Stiening, R., et al. 2006, AJ, 131, 1163

Wilking, B. A., Greene, T. P., \& Meyer, M. R. 1999, AJ, 117, 469

Witham, A. R., Knigge, C., Drew, J. E., et al. 2008, MNRAS, 384, 1277

Zapatero Osorio, M. R., Béjar, V. J. S., Pavlenko, Y., et al. 2002a, A\&A, 384, 937

Zapatero Osorio, M. R., Béjar, V. J. S., Martín, E. L., Barrado y Navascués, D., \& Rebolo, R. 2002b, ApJ, 569, L99 